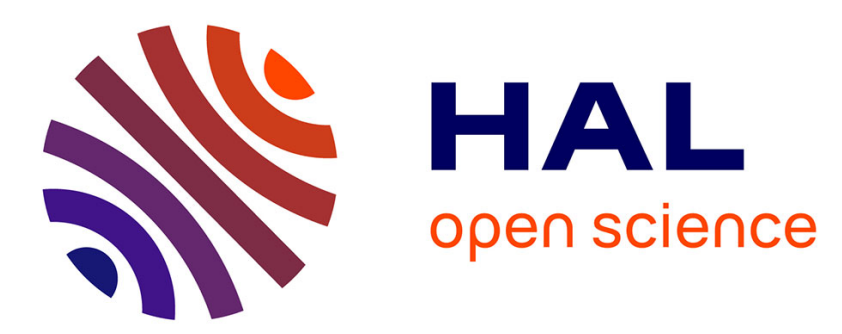

\title{
Patterns formations in a diffusive ratio-dependent predator-prey model of interacting populations
}

\author{
B.I. Camara, M. Haque, H. Mokrani
}

\section{To cite this version:}

B.I. Camara, M. Haque, H. Mokrani. Patterns formations in a diffusive ratio-dependent predator-prey model of interacting populations. Physica A: Statistical Mechanics and its Applications, 2016, 461, pp.374-383. 10.1016/j.physa.2016.05.054 . hal-03208949

\section{HAL Id: hal-03208949 \\ https://hal.univ-lorraine.fr/hal-03208949}

Submitted on 27 Apr 2021

HAL is a multi-disciplinary open access archive for the deposit and dissemination of scientific research documents, whether they are published or not. The documents may come from teaching and research institutions in France or abroad, or from public or private research centers.
L'archive ouverte pluridisciplinaire HAL, est destinée au dépôt et à la diffusion de documents scientifiques de niveau recherche, publiés ou non, émanant des établissements d'enseignement et de recherche français ou étrangers, des laboratoires publics ou privés. 


\title{
Pattern formation in a diffusive ratio-dependent predator-prey model of interacting populations
}

\author{
B. I. Camara ${ }^{a, *}$, M. Haque ${ }^{b}$, H. Mokranic ${ }^{\mathrm{c}}$ \\ ${ }^{a}$ Université de Lorraine - CNRS UMR 7360 \\ Laboratoire Interdisciplinaire des Environnements Continentaux \\ Campus Bridoux - 8 Rue du Général Delestraint, 57070 Metz, France \\ ${ }^{b}$ Nottingham University Hospitals NHS \\ University Division of Anaesthesia Intensive Care, \\ Queen's Medical Centre Campus, \\ Derby Road, Nottingham, U.K. \\ ${ }^{c}$ Université de Rouen - CNRS UMR 6085 \\ Laboratoire de Mathématiques Raphaël Salem \\ Avenue de l'Université, '76801 Saint-Étienne-du-Rouvray, France
}

\begin{abstract}
The present investigation deals with the analysis of the spatial pattern formation of a diffusive predator-prey system with ratio-dependent functional response involving the influence of intra-species competition among predators within two-dimensional space.

The appropriate condition of Turing instability around the interior equilibrium point of the present model has been determined.

The emergence of complex patterns in the diffusive predator-prey model is illustrated through numerical simulations. These results are based on the existence of bifurcations of higher codimension such as Turing-Hopf, TuringSaddle-node, Turing-Transcritical bifurcation, and the codimension-3 TuringTakens-Bogdanov bifurcation. The paper concludes with discussions of our results in ecology.
\end{abstract}

Keywords: Intra-species competition, Pattern formation, Turing-Hopf bifurcation, Turing-Saddle-node, Turing-Transcritical bifurcation, Turing-Hopf-Andronov bifurcation

\footnotetext{
*Corresponding author

Email addresses: baba-issa.camara@univ-lorraine.fr (B. I. Camara), Mainul.Haque@nottingham.ac.uk (M. Haque), houdamokrani@yahoo.fr (H. Mokrani )
} 


\section{Introduction}

One of the most challenging aspects of studying ecological systems is to find out how organisms interact with each other in a natural environment. These interactions can take place directly between 2 or more species like predation, symbioses or competition. Pattern formation has been a major interest of theorists and experimentalists, and it is generally thought that spatial patterns form as a consequence of the interplay of various physical and biological processes [17]. Turing [25] showed how the coupling of reaction and diffusion can induce pattern formation. Since then, the mechanism responsible for the spontaneous generation of spatial patterns through biological or chemical interactions has been called diffusion instability. In this process spatial patterns arise not from inhomogeneity of initial or boundary conditions, but purely from the dynamics of the system, i.e. from the interaction of nonlinear reactions of growth processes and diffusion.

Segel and Jackson [21] were the first to apply Turing's idea to a problem in population dynamics: The dissipative instability in the predator-prey interaction of phytoplankton and herbivorous copepods with higher herbivore motility.

The study of biological pattern formation has gained popularity. Recently particular interests have been on the impact of environmental changes $[4$, 16, 17]. The dynamic relationships between species and their complex properties are at heart of many important ecological and biological processes. Predator-prey dynamics are a classic and relatively well-studied example of interactions.

Camara and Aziz-Alaoui [6] consider a predator-prey system modeled by a reaction-diffusion equation. It incorporates the Holling-type II and a modified Leslie-Gower functional responses. Their paper addresses the analysis of the global stability of the endemic equilibrium, the bifurcations and spatiotemporal dynamics of a system of this type. In their paper [7], they focus on spatiotemporal patterns formation. They showed how diffusion affects the stability of predator-prey positive equilibrium and derive the conditions for Hopf and Turing bifurcation in the spatial domain. Her findings suggest that Hopf instability leads to the formation of symmetric labirynth and Turing instability destroys a spatiotemporal chaos and leads to the formation of labirynth pattern. 
Camara $[7,8]$ considered a system of reaction-diffusion equations to model the spatiotemporal dynamics of three species linked by a trophic food chain. Through the analysis of bifurcations, pattern complexity was explored by determining the critical parameter values, which lead to ecosystem changes in a bottom-up scenario of control.

Feudel et al. [10] studied spatiotemporal patterns in marine systems as a result of the interaction of population dynamics with physical transport processes. These physical transport processes can be either diffusion processes in marine sediments or in the water column. They have shown that Turing patterns can occur which yield heterogeneous spatial patterns of the species. Upadhyay and Thakur [26] performed spatiotemporal dynamics and pattern formation in a model aquatic system with Beddington-DeAngelis type functional response in both homogeneous and heterogeneous environments. Their model incorporates the spatial heterogeneity in the growth rate of phytoplankton population and the effect of fish predation in the model system which follows Holling type III functional response. Her findings suggest that The Turing instability destroys a spatiotemporal chaos and leads to the formation of wavy structure. Alonso et al. et al. [2] pointed out that mutual interference between predators could give rise to Turing spatial patterns under certain conditions of trophic interaction. These non-uniform spatial distributions arise through diffusion-driven instabilities. Haque [12] showed, analytically that the difference pointed out by Alonso et al. [2] between prey dependent and ratio dependent predator-prey model is no longer valid when competition among the predator population is being incorporated. Observing the importance of ratio-dependent functional response in the predator-prey model, Haque [12] modified the classical Bazykin's [5] model by taking the ratio-dependent predation term into account instead of the prey-dependent predation term when a predator experiences serious hunting process, and his model takes the following form:

$$
\begin{aligned}
\frac{d u}{d t} & =r u\left(1-\frac{u}{k}\right)-\frac{m u v}{a u+v}=F_{1}(u, v), \\
\frac{d v}{d t} & =\frac{e m u v}{a u+v}-d v-h v^{2}=F_{2}(u, v), \\
u(0) & =u_{0}>0, \quad v(0)=v_{0}>0,
\end{aligned}
$$

where $v(t), v(t)$ stands for the prey and predator density, respectively, at time $t . r, k, m, a, e, d$, are positive constants that stand for prey intrinsic growth rate, carrying capacity of the environment, consumption rate, prey 
saturation constant, conversion rate, predator death rate, respectively. The parameter $h$ measures the predator intra-specific competition.

Models with intra-species competition have been extensively studied in literature $[5,4,12,3]$.

In the evolutionary process of population species, individuals do not remain fixed in space and their spatial distributions change continuously due to the impact of many factors. Therefore, different spatial effects have been introduced into prey-predator model $(1)$, such as diffusion $[14,11]$. In the present investigation we focus on a special class of spatio-temporal patterns that are very likely to be found in the neighborhood of Turing-Hopf bifurcations. In this way there has been a growing understanding, what transitions between different dynamical regimes arising as a result of perturbation of the system's parameters.

The paper is organized as follows: In Section 2, the analytical behavior of the temporal model around the positive interior equilibrium is briefly studied. In section 3, with respect to some parameters we perform a series of simulation to illustrate the emergence of spatiotemporal patterns. The obtained results show that the modeling by both intra-specific competition and diffusion is useful for studying the dynamic complexity of ecosystems. Finally, some conclusions and comments based on numerical simulations exhibiting quantitative response of the system are included in Section 4.

\section{Model formulations}

In the current paper, the spatiotemporal behaviors of the prey-predator system (1) are described under uniform environment. In order to consider this, we incorporate into model (1) spatial effects by deriving, the following reaction-diffusion model

$$
\begin{aligned}
& \frac{\partial u}{d t}=F_{1}(u, v)+D_{u} \Delta u \\
& \frac{\partial v}{d t}=F_{2}(u, v)+D_{v} \Delta v
\end{aligned}
$$

with initial condition

$$
u(x, y, 0)>0, v(x, y, 0)>0, \text { for }(x, y) \in[0, L] \times[0, L],
$$

and boundary conditions

$$
(\mathbf{n} . \nabla)(u, v)^{T}=0, \quad \text { on } \quad \partial \bar{\Omega},
$$


where $\partial \bar{\Omega}$ is the closed boundary of the reaction-diffusion domain $\bar{\Omega}$ and $\mathbf{n}$ is the outward unit normal vector to $\partial \bar{\Omega}$. The zero flux boundary conditions imply that no species enter or leave the defined environment. $D_{u}$ and $D_{v}$ are the diffusion coefficients of prey and predator respectively.

Haque $[12,14]$ obtained that the system (1) possesses three steady states:

$$
E_{0}(0,0), E_{1}(k, 0), E_{2}\left(u^{*}, v^{*}\right) .
$$

where $\left(u^{*}, v^{*}\right)$ are given in A.2.

The equilibrium point $E_{0}$ corresponds to absence of both species, $E_{1}$ means the extinct of prey, and $E_{2}$ corresponds to coexistence of the two species. The first two equilibria $E_{0}$ and $E_{1}$ obviously exist. From Ref. [13], we know that there exists a real positive interior equilibrium point $E_{2}$ if $\psi_{2}>0$. The stability analysis of the system (2) around $E_{2}$ has been carried out in $[12,11]$.

\subsection{The behavior of the system around the interior equilibrium $E_{2}$}

We derive the conditions for Turing-Saddle-node, Turing-Hopf, and TuringBogdanov, bifurcation around $E_{2}$

Theorem 1. (i) The system (1) experiences a saddle-node bifurcation around $E_{2}$ at $m=m^{[s n]}$, where $m^{[s n]}=\frac{r\left(k^{2} a^{2} h^{2}+2 k \theta_{1} h a-d^{2}-2 d \theta_{1}\right)}{4 k \theta_{1} h a}$, where $\theta_{1}$ is given in Appendix A.1.

(ii) The system (1) enters into a Hopf-bifurcation around $E_{2}$ at $m=m^{[h b]}$, where $m^{[h b]}$ is given in Appendix A.1.

(iii) The system (1) enters into a Takens-Bogdanov bifurcation around $E_{2}$ at $m=m^{[t b]}$ for $d=d^{[t b]}$; where $m^{[t b]}$ and $d=d^{[t b]}$ are given in Appendix A.1.

\section{Simulations of patterns from bifurcations around $E_{2}$}

In this section, we find a general space where all kinds of bifurcations mentioned above could be experienced by the system (2a)- (2b). We took a set of admissible parameter values:

$$
h=0.0063, \quad k=16.0, \quad a=1.453, \quad e=0.32, \quad d=0.187
$$

When prey intrinsic growth rate $r$ and predator consumption rates $m$ vary, model system (2a)-(2b), describing only the temporal dynamics of predatorprey interactions, leads to several local bifurcations around equilibrium $E_{2}$. 
In Fig. 1, blue area corresponds to the values of $r$ and $m$ for which $E_{2}$ does not exist. The light yellow (respectively dark yellow) color represents the values of $r$ and $m$ for which $E_{2}$ is a stable node (respectively a stable spiral). The light red (respectively dark red ) color represents the values of $r$ and $m$ for which $E_{2}$ is an unstable node (respectively an unstable spiral).

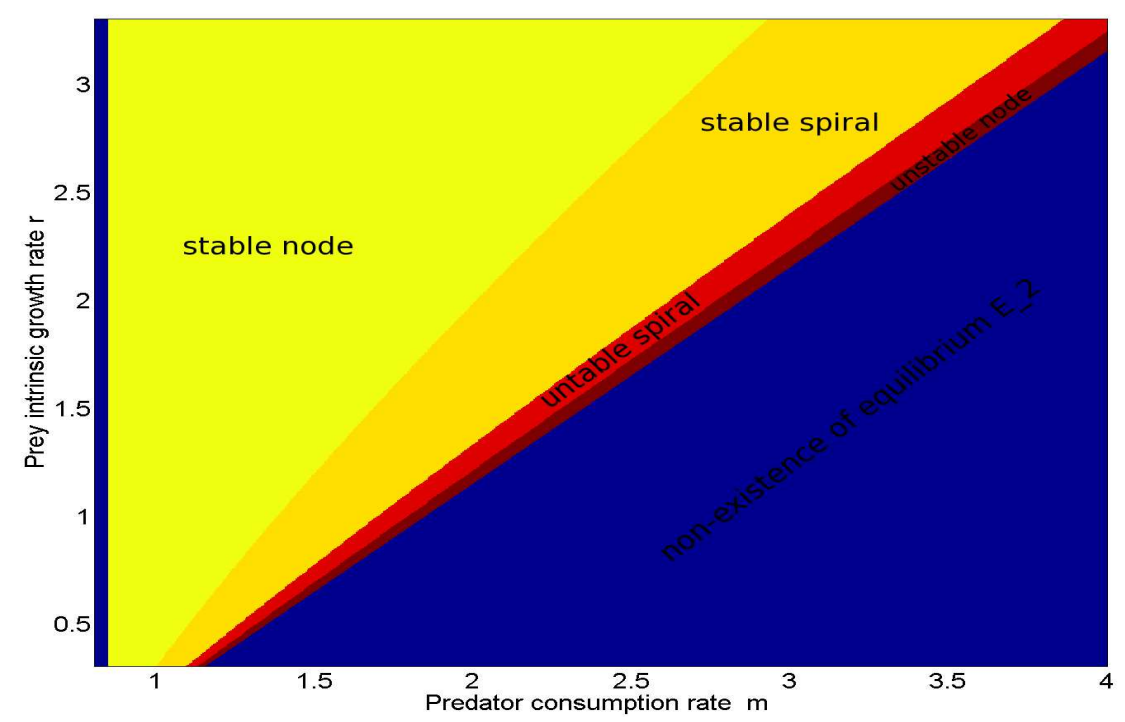

Fig. 1: Local bifurcation of model (1) around the interior equilibrium $E_{2}$ when prey intrinsic growth rate $r$ and predator consumption rates $m$ vary with parameter values: $h=0.0063, k=16.0, a=1.453, e=0.32, d=0.187$.

\subsection{Patterns in an excitable medium}

Here we take into account the spatial dimension by considering the model (2) defined in the square numerical domain $\Omega=[0,600] \times[0,600]$ with zero-flux boundary condition and by varying $m$ and $r$. We assume that the two populations have the same diffusion coefficients $\left(D_{u}=D_{v}=0.82\right)$, and we fix the other parameters as follows,

$$
h=0.0063, \quad k=16.0, \quad a=1.453, \quad e=0.32, \quad d=0.187 .
$$

Values of $m$ and $r$ in yellow area of Fig. 1 correspond to local stability of equilibrium $E_{2}$ for PDE (2). However in red area, equilibrium $E_{2}$ is also 
unstable for PDE (2) and this leads to complex pattern formation. In order to study numerically these complex patterns and bifurcations of model (2) around equilibrium $E_{2}$, we fix the initial population densities (at time $t=0$ ) close to equilibrium $E_{2}$ as follows,

$$
\begin{aligned}
& u(x, y, 0)=u^{*}, \\
& v(x, y, 0)=v^{*}+0.05(w-0.5),
\end{aligned}
$$

where $w$ is randomly generated and follows a $[0,1]$ uniform distribution. In Fig. 2, the spatiotemporal model (2) exhibits spiral waves when predator and prey have the same diffusion rate. In this figure, $m$ and $r$ are varying in red area of Fig. 1, and the other parameters are fixed as in (5). In Fig. 2(A1)(A2), $m$ and $r$ are in the dark red area of Fig. 1, and in Fig. 2(B1)-(B2), $m$ and $r$ are in the light red area of Fig. 1. These spiral waves emerge from a combination of nonlinear neighborhood interactions and local dispersal and are a characteristic of excitable media. In fact, spatiotemporal model (2) shows excitable kinetics meaning that small perturbations from homogeneous equilibria are damped down, but if these perturbations go beyond a critical threshold they are amplified [23]. These spiral waves are not stationary and change in time and space.

\subsection{Patterns in non-excitable medium}

In this section, using numerical methods, we perform numerical simulations of the system (2) in a two-dimensional space and illustrate that reactiondiffusion induce spatial patterns.

When prey intrinsic growth rate $r$ and predator consumption rates $m$ are in the yellow area of Fig. 1, the interior equilibrium $E_{2}$ is locally stable for model (2). In fact, when predator and prey have the same diffusion rate, $E_{2}$ is also stable for model the spatiotemporal model (2) and there is no pattern formation. The instability leading to the pattern formation, known as diffusion-driven instability or Turing instability is caused by the fact that the predator diffuses faster than the prey. When we fix $D_{v}=1.95, \frac{D_{v}}{D_{u}}>60$ and the other parameters are fixed as in (5). Light blue area of Fig. 3(A) correspond to values of $m$ and $r$ leading to Turing instability when $\frac{D_{v}}{D_{u}}>60$. As we can see in Fig. 3(B), a spontaneous stationary pattern emerges. However when the intrinsic growth rate $r$ and the rate of competition $h$ vary, 

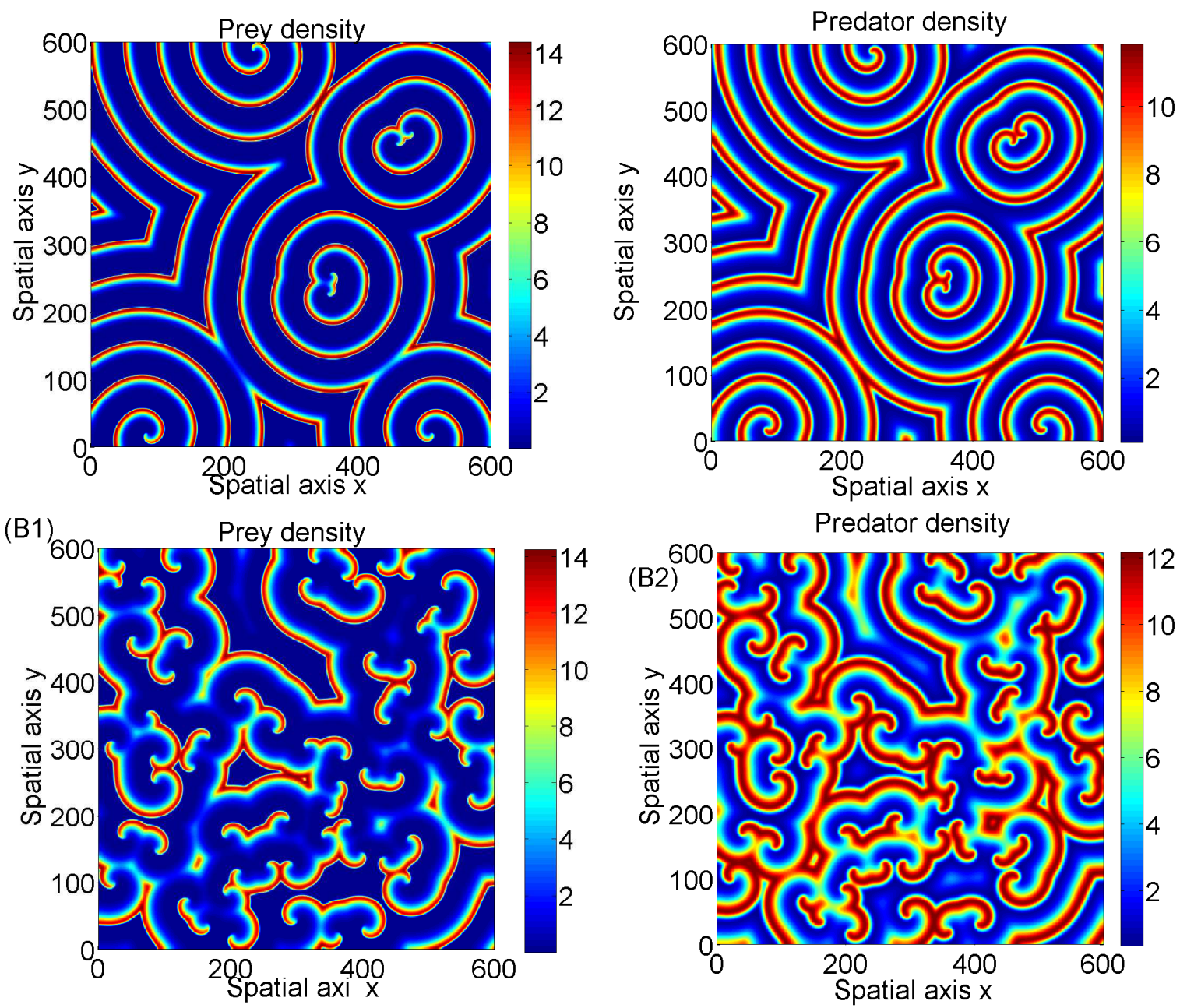

Fig. 2: Emergence of spiral waves in excitable media for $D_{u}=D_{v}=0.82$. Figures 2 (A1)-(A2): $m=3.495$ and $r=2.71807$. Figures 2 (B1)-(B2): $m=3.495$ and $r=2.785$.

the region corresponding to non-excitable media is bounded by three bifurcations: Turing bifurcation, Turing-Saddle-Node bifurcation and TuringTranscritical bifurcation (see Fig. 4). The occurrence of saddle-node bifurcation and transcritical bifurcation is independent of the spatial dimension of the model. In fact these two bifurcations occur when two isoclines of different natures, initially disjointed become tangent and intersect at the bifurcation point. Then, two equilibrium points appear, the unstable and the other sta- 
ble $\left(E_{2}\right.$ in system 1$)$. However, the diffusion process generates the instability of equilibrium $E_{2}$ for system 2 . Thus, the combination of saddle-node bifurcation or transcritical bifurcation with diffusion process (parameter values are in the region of bifurcations Fig. 4), leads to Turing stationary patterns.
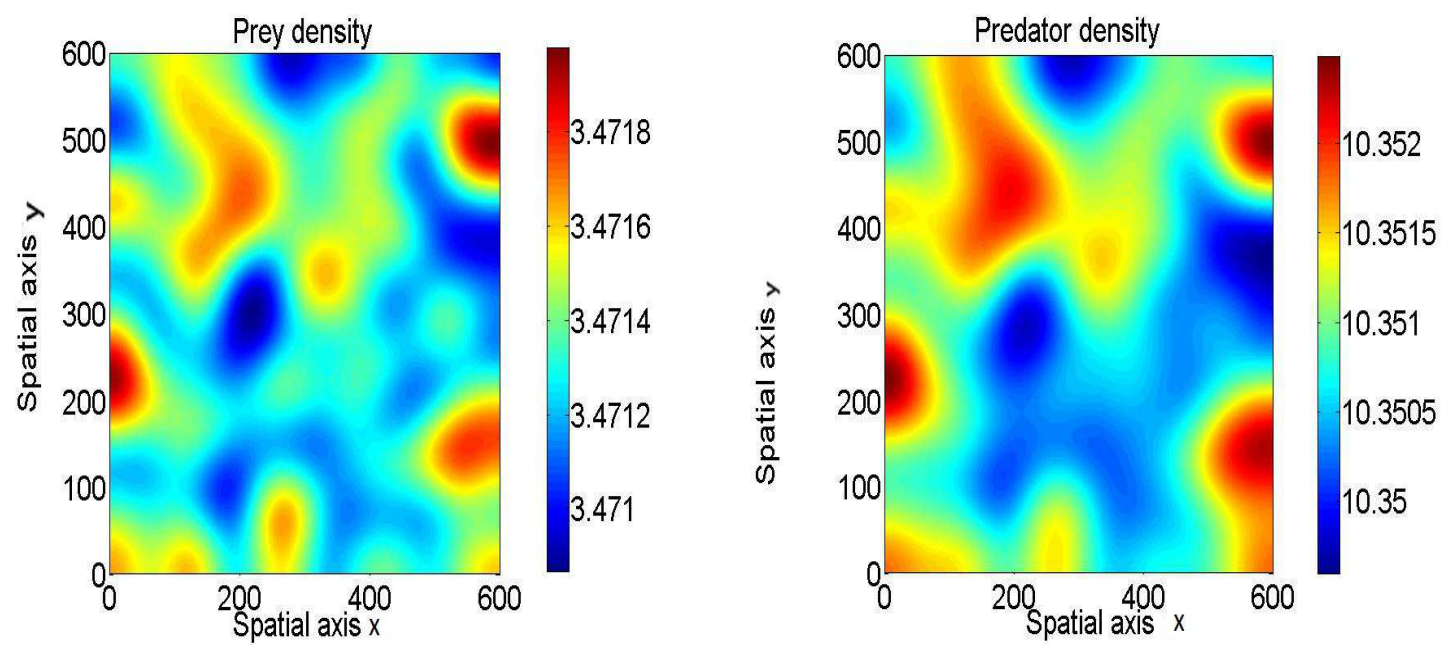

Fig. 3: Zone of Turing instabilities when $\frac{D_{v}}{D_{u}}>60, h=0.0063, k=16.0, a=1.453$, $e=0.32$ and $d=0.187$.

\subsection{Patterns in interface between excitable and non-excitable medium}

Fig. 5 represents according to model parameters, the interface between the excitable and non-excitable media. When the prey intrinsic growth rate $r$, predator consumption rate $m$ and intra-specific competition $h$ vary together, Fig. 5(A) shows the correlation of these parameters with the emergence of Turing-Hopf-Andronov bifurcations. Thus, the prey intrinsic growth rate $r$ and the predator consumption rate $m$ positively influence the emergence of a Turing-Hopf- Andronov bifurcation while the competition has no effect on the emergence of such a bifurcation. However, Fig. 5(B) illustrate the correlation between the emergence of Turing-Hopf-Andronov bifurcation and the joint variation of predator consumption rate $m$, intra-specific competition $h$ and the ratio of diffusion $\frac{D_{v}}{D_{u}}$. Thus, we have in Fig. 5(B), the 


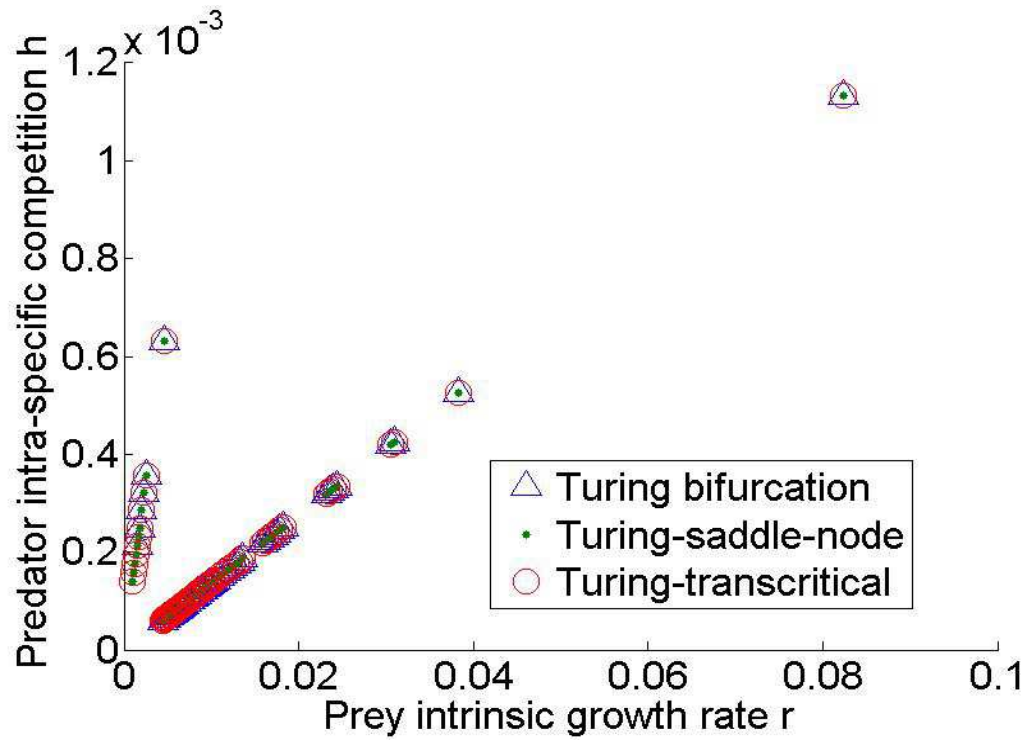

Fig. 4: Bifurcations of Turing, Turing-Saddle-Node and Turing-Transcritical, when intrinsic growth rate $r$ and competition rate $h$ vary, with $D_{u}=0.02$ and $D_{v}=0.055$

competition rate $h$ is negatively correlated, the ratio of diffusion $\frac{D_{v}}{D_{u}}$ is positively correlated while the predator consumption rate $m$ is not correlated with the emergence of a Turing-Hopf-Andronov bifurcation. One characteristic of a Turing-Hopf-Andronov bifurcation is the coexistence of Turing wavelength and the wavelength of a traveling wave. For long Turing wavelengths, short wavelength traveling waves appear on the Turing structures. Several oscillatory Turing patterns of this phenomenon have been shown in Ref. [28], where the Turing wavelength is about 4 times longer than that of the traveling waves. However, if the wavelength of the Turing pattern is short compared with that of the wave mode, modulated patterns [27]. This behavior indicates that nonlinear effects arising from the interaction between the Turing and wave modes suppress the pure Turing mode and leads to the emergence of oscillatory patterns.

Fig. 5 shows modulated Turing structures develop from a standing wave pattern. First, the initial unstable standing wave gives way to an almost uniform steady state, which is gradually converted into a Turing pattern (Fig. 5(A)). This pattern remains almost unchanged for a significant pe- 
riod of time after which modulation, first by standing and then by traveling waves, becomes apparent (Fig. 5(B)). However, with a changing parameter value $r=2.05311579, \quad m=2.67, \quad D_{u}=0.02, \quad D_{v}=0.055$, the oscillatory state undergoes a series of bifurcation and becomes chaotic (Fig. 6).

Fig. 6 is a spatiotemporal chaos from Turing-Hopf-Andronov bifurcation. This figure shows that an interaction between Turing and wave modes may lead to the lost of Turing structures and spiral wave patterns, making chaotic patterns emerge.
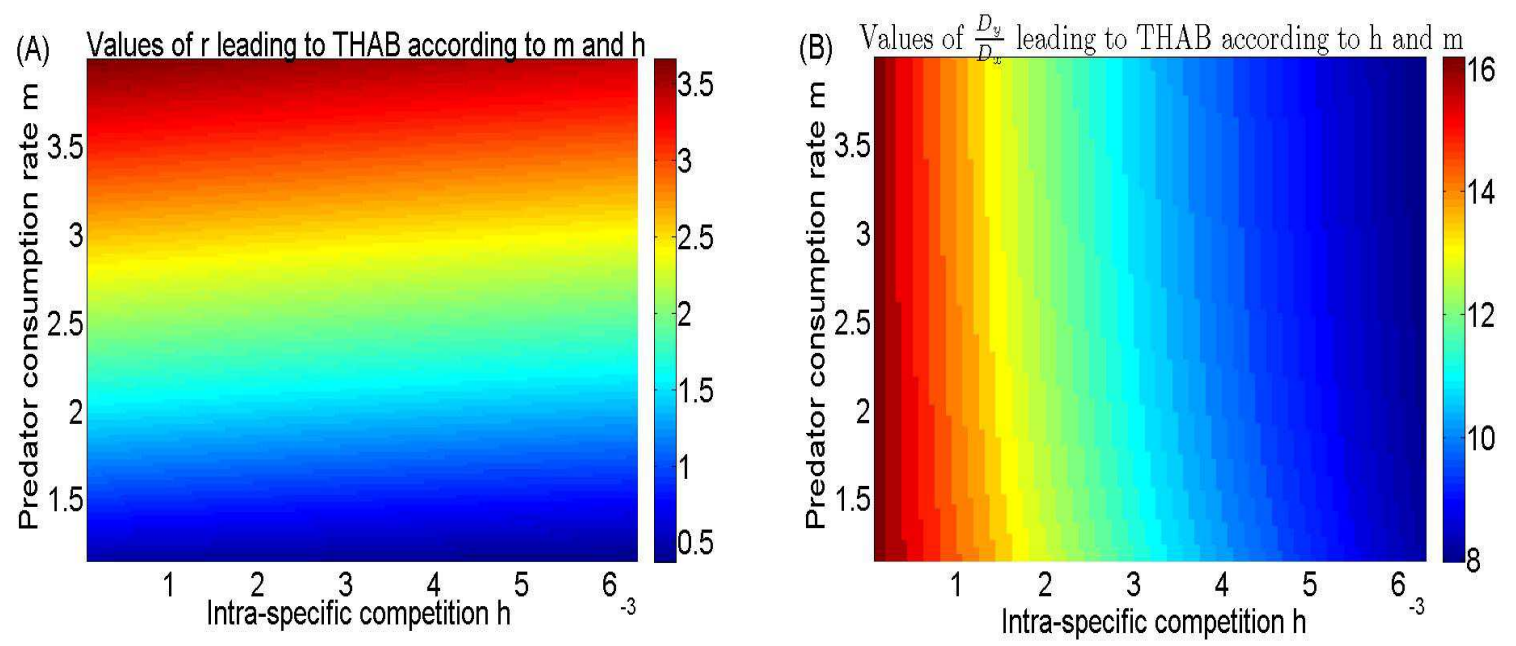

Fig. 5: Relationships between the model parameters leading to instability of Turing Hopf Andronov, with $e=0.32, d=0.137, \quad h \in] 0,6[, \quad m \in] 0,4\left[\right.$. In $(\mathrm{A}): D_{u}=0.02, D_{v}=$ 0.055 , in $(\mathrm{B}): r=2.05311579$.

\section{Conclusion and discussion}

The present investigation deals with the analysis of the spatial pattern formation of a diffusive predator-prey system with ratio-dependent functional response involving the influence of intra-species competition among predators within two-dimensional space. There are many ways in which a particular type of intra-specific competition might be considered advantageous or disadvantageous to a population. In this regard most references in the literature study the stability versus instability of a positive equilibrium $[15,19]$. 

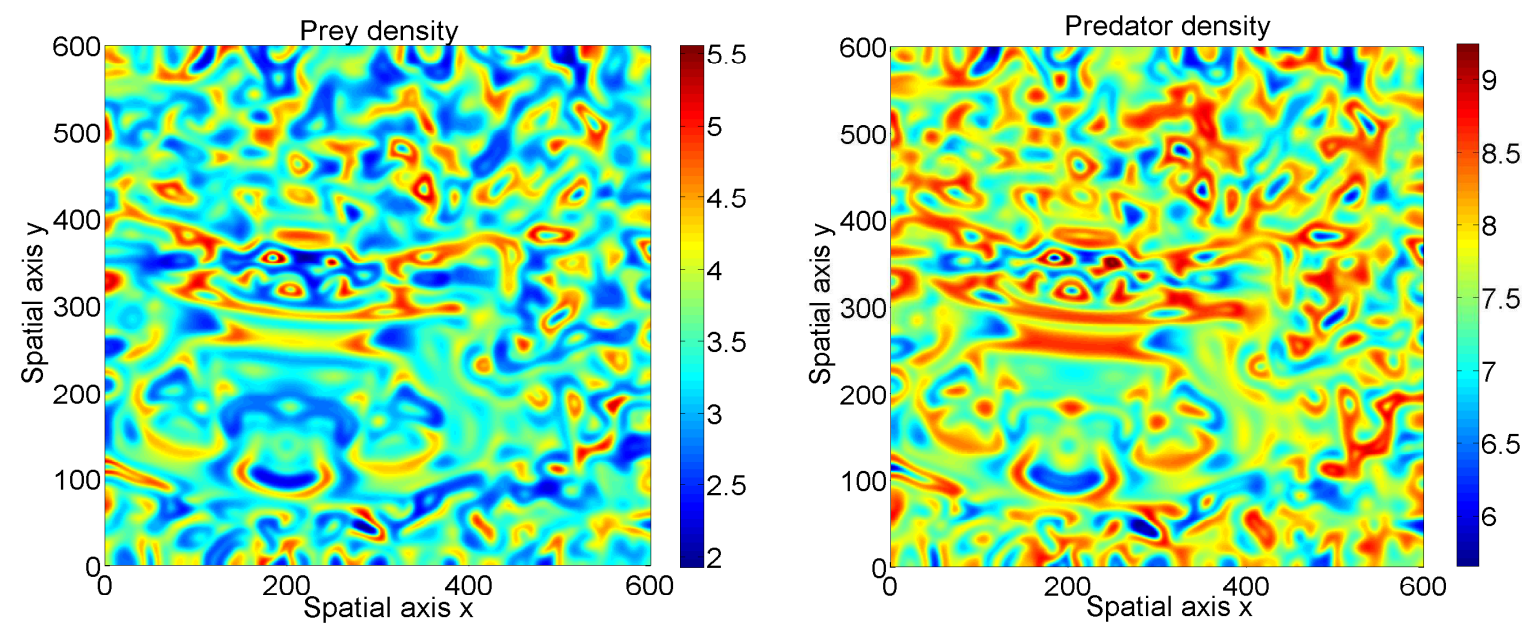

Fig. 6: Emergence of spatio-temporal chaos from Turing-Hopf-Andronov bifurcation, with $a=1.453, \quad e=0.32, \quad d=0.187 \quad h=0.0063, \quad r=2.05311579, \quad m=2.67$.

Based on the bifurcation conditions, we simulate numerically the spatial pattern formation, the evolution process of the system near the interior equilibrium. We find in Fig. 2 that Hopf instability leads to the formation of spiral wave patterns and show in Fig. 3, patterns from Turing instability. Our investigations reveal correlation between parameters and the emergence of Turing-Hopf-Andronov bifurcations. We obtain interacting spiral wave patterns as chaotic pattern for the parameter values outside the Turing domain. We also discover that the Turing-Hopf-Andronov bifurcation can drive spatiotemporal chaotic dynamics (Fig. 6). These chaotic patterns emerge from nonlinear interaction Turing mode and wave mode. However, it requires that the wavelength of the mode wave to be greater than that of Turing mode. This emerging patterns or modulated patterns induce a lost of Turing instability and spiral pattern. Interactions between Turing mode and wave mode can also induce modulated standing waves [28].

In ecology, oscillations are associated with destabilization. The reason is that extinction of the population due to natural fluctuations becomes very likely when the oscillation drives the population to low abundances [22]. For a population, it has been shown that chaotic dynamics can be of advantage in order to stabilize the whole population. Consequently it reduces the probability of a global extinction [1]. The mathematical analysis combined 
with numerical investigation reveals the complexity of the structural organization of interacting populations. A change of the model parameter values can categorically infer the structure of this organization [8]. The stability analysis of structures can prevent absolute changes. As in [9], knowledge of the distribution shape can be an indicator of environmental quality.

\section{References}

[1] J. C. Allen, W.M. Schaffer, D. Rosko, Chaos reduces species extinction by amplifying local-population noise. Nature 364 (1993) 229-232.

[2] D. Alonso, F. Bartumeus, J. Catalan, Mutual interference between predators can give rise to Turing spatial patterns. Ecology (2002) 83 $28-34$.

[3] N. Apreutesei, A. Ducrot, V. Volpert, Competition of Species with IntraSpecific Competition. Math. Model. Nat. Phenom. 3(4) (2008) 1-27.

[4] M. Baurmanna, T. Gross, U. Feudela, Instabilities in spatially extended predator-prey systems: Spatio-temporal patterns in the neighborhood of Turing-Hopf bifurcations. J. Theoret. Biol. 245 (2007) 220-229.

[5] A.D. Bazykin, Nonlinear dynamics of interacting populations, World Scientific Series on Nonlinear Science Series A: Volume 11, 1998.

[6] B.I. Camara, M.A. Aziz-Alaoui, Dynamics of a Predator-prey model with diffusion. Dynamics of Continuous Discrete and Impulsive Systems, Series A : Mathematical Analysis 15 (2008) 897-906.

[7] B.I. Camara, M.A. Aziz-Alaoui, Turing and Hopf Patterns Formation in a Predator-Prey Model with Leslie-Gower-Type Functional Response. Dynamics of Continuous, Discrete and Impulsive Systems, series B 16(4) (2009) 479-488.

[8] B.I. Camara, Waves analysis and spatiotemporal pattern formation of an ecosystem model. Nonlinear Anal. Real World Appl. 12 (2011) 25112528.

[9] B.I. Camara, Food web complexity analysis: effects of ecosystem changes. Nonlinear Dynamics 73 (2013) 1783-1794. 
[10] U. Feudel, M. Baurmann, T. Gross, Spatio-temporal patterns in simple models of marine systems. Geophysical Research Abstracts 11 EGU General Assembly, 2009, pp. 13135.

[11] L.N. Guin, P.K. Mandal, Spatiotemporal dynamics of reaction-diffusion models of interacting populations. Applied Mathematical Modelling 38 (2014) 4417-4427.

[12] M. Haque, Ratio-Dependent Predator-Prey Models of Interacting Populations. Bull. Math. Biol. 71 (2009) 430-452.

[13] M. Haque, A Detailed Study of the Beddington-DeAngelis predator-prey Model. Math. Biosc. 234 (2011) 1-16.

[14] M. Haque, Existence of complex patterns in the Beddington-DeAngelis predator-prey model. Math. Biosc. 239 (2012) 179-190.

[15] A. Lomnicki, S. Stanislaw, Resource partitioning and population stability under exploitation competition. J. Theor. Biol. 132 (1988) 119-120.

[16] H. Malchow, S.V. Petrovskii, E. Venturino, Spatiotemporal Patterns in Ecology and Epidemiology: Theory, Models, and Simulations. Chapman and Hall /CRC Press, London, 2008.

[17] A.B. Medvinsky, S.V. Petrovskii, I.A. Tikhonova, H. Malchow, B.L. Li, Spatiotemporal complexity of plankton and fish dynamics. SIAM Rev. 44 (2002) 311-370.

[18] J.D. Murray, Mathematical Biology: Spatial models and biomedical applications, Springer Verlag, 2003.

[19] A.R. Rogers, Population dynamics under exploitation competition, J. Theor. Biol. 119 (1986) 363-368.

[20] W. Rudin, Principles of mathematical analysis, McGraw-Hill, New York, 1976.

[21] L.A. Segel, J.L. Jackson, Dissipative structure: an explanation and an ecological example. J. Theor. Biol. 37 (1972) 545-559. 
[22] A.O. Sheltona, M. Marc Mangel, Fluctuations of fish populations and the magnifying effects of fishing. Proc. Natl. Acad. Sci. 108 (2011) 70757080 .

[23] R.V. Solé, J. Bascompte, Self-organization in Complex Ecosystems, Princeton Univ. Press, Princeton, 2006.

[24] J. Sotomayor, Generic bifurcations of dynamical systems. In: Proc. Sympos. on Dynamical systems, Univ. Bahia, Salvador, Academic Press, New York, 1973, pp. 561-582.

[25] A.M. Turing, Chemical basis of morphogenesis. Phil. Trans. R. Soc. B 327 (1952) 37-72.

[26] R.K. Upadhyay, N.K. Thakur, Diffusion-Driven Instabilities and Spatio-Temporal Patterns in AN Aquatic predator-prey System with Beddington-DeAngelis Type functional response. International Journal of Bifurcation and Chaos 21 (2011) 1-22.

[27] L. Yang, M. Dolnik, A.M. Zhabotinsky, I.R. Epstein, Pattern formation arising from interactions between Turing and wave instabilities. Journal of chemical physics 117 (2002) 1-7.

[28] L. Yang, I.R. Epstein, Oscillatory Turing patterns in reaction-diffusion systems with two coupled layers. Physical review letters 90 (2003) 1-4.

\section{Appendix A. Turing instability Conditions}

the Jacobian matrix $J$ of the system (1) is given by

$$
J=\left[\begin{array}{cc}
r\left(1-\frac{u}{k}\right)-\frac{r u}{k}-\frac{m u}{a u+v}+\frac{m a u v}{(a u+v)^{2}} & -\frac{m u}{a u+v}+\frac{m u v}{(a u+v)^{2}} \\
\frac{e m v}{a u+v}-\frac{e m a u v}{(a u+v)^{2}} & \frac{e m u}{a u+v}-\frac{e m u v}{(a u+v)^{2}}-d-2 h v
\end{array}\right]=\left(\zeta_{i j}\right)_{2 \times 2} .
$$

Using the Sotomayor theorem [24], and Murray [18], we have the following theorem: 
Theorem 2. Suppose $\frac{\partial W}{\partial t}=F(W, \lambda)+\mathcal{D} \nabla W$, where $F(W, \lambda)=\left(F_{1}, F_{2}\right)^{T}=$ $0, W=(u, v)^{T}, \mathcal{D}=\left(\begin{array}{cc}D_{u} & 0 \\ 0 & D_{v}\end{array}\right)$ and $\lambda$ is taken as a bifurcation parameter. Let $J \equiv A F(W, \lambda)=\zeta_{2 \times 2}$ be the Jacobian matrix of this system. If $U$ and $V$ are two eigenvectors corresponding to a zero eigenvalue of the matrix $J$ and its transpose $J^{T}$ respectively, then system experiences

The necessary and sufficient conditions for Turing instability (TI) to arise as follows:

Turing instability (TI): $\operatorname{tr} J<0, \operatorname{det} J>0, D_{v} \zeta_{11}^{[2]}+D_{u} \zeta_{22}^{[2]}>0$, $\left(D_{v} \zeta_{11}^{[2]}+D_{u} \zeta_{22}^{[2]}\right)^{2}-4 D_{u} D_{v} \operatorname{det} J>0$

Turing-Saddle-node(TSNB): $\operatorname{tr} J<0, \operatorname{det} J=0, D_{v} \zeta_{11}+D_{u} \zeta_{22}>0, D_{v} \neq D_{u}$ $V^{T}\left[F_{\lambda}(W, \lambda)\right] \neq 0, V^{T}\left[A^{2} F(W, \lambda)(U, U)\right] \neq 0$;

Turing-Transcritical(TTCB): $\quad \operatorname{tr} J<0$, det $J=0, D_{v} \zeta_{11}+D_{u} \zeta_{22}>0, D_{v} \neq D_{u}$, $V^{T}\left[F_{\lambda}(W, \lambda)\right]=0, V^{T}\left[A F_{\lambda}(W, \lambda) U\right] \neq 0$, $V^{T}\left[A^{2} F(W, \lambda)(U, U)\right] \neq 0$;

Turing-Hopf-Andronov(THAB): $\quad \operatorname{tr} J=0, \operatorname{det} J>0, \zeta_{22}\left(D_{u}-D_{v}\right)>0$, $\left(\zeta_{22}\left(D_{u}-D_{v}\right)\right)^{2}>4 D_{u} D_{v} \operatorname{det} J, D_{v} \neq D_{v}$;

Turing-Bogdanov-Takens(TBTB): $\quad \operatorname{tr} J=0, \operatorname{det} J=0, \zeta_{22}\left(D_{u}-D_{v}\right)>0$;

bifurcation around $W$, where $A F_{\lambda}=\frac{\partial(A F)}{\partial \lambda}$; and $A^{2} F(W, \lambda)(U, U), A^{3} F(X, \lambda)(U, U, U)$ are defined in Appendix A.2.

Appendix A.1. Expressions for $\theta_{1}, \theta_{2}, m^{[t b]}, d=d^{[t b]}$ and $\theta_{3}$.

$\theta_{1}$ is given by

$$
\theta_{1}=-\frac{a^{2} d h k+e r d \pm \sqrt{a^{4} d^{2} h^{2} k^{2}+d^{2} a^{2} e h k r+r k^{3} a^{4} h^{3} e+r^{2} k^{2} a^{2} h^{2} e^{2}}}{2\left(a^{2} h k+e r\right)}
$$

$m^{[h b]}$ is given by 


$$
\begin{aligned}
m^{[h b]}= & \left(2 a^{3} h^{2} k^{2} \theta_{3} d-2 a^{3} h^{2} k^{2} \theta_{2} r-2 a^{3} h k^{2} r d+2 a^{3} h k^{2} d^{2}+a^{2} e \theta_{2}{ }^{2} k^{2} h^{3}+a^{2} e h^{2} k^{2} \theta_{2} r\right. \\
& -2 h r^{2} k^{2} a^{2} e-a^{2} e h k^{2} d^{2}+3 a^{2} e h k^{2} r d+4 a^{2} \theta_{2}^{2} r k h^{2}+6 a^{2} \theta_{2} d r k h+2 r d^{2} k a^{2} \\
& +2 a e^{2} h^{2} k^{2} \theta_{2} r+2 r^{2} e^{2} k^{2} a h-6 a e \theta_{2}^{2} r k h^{2}-5 a e \theta_{2} d r k h-a e h k \theta_{2} r^{2}-r e d^{2} k a \\
& +a e k d r^{2}+4 e^{2} \theta_{2}{ }^{2} r k h^{2}+2 e^{2} h k \theta_{2} r^{2}+2 e^{2} \theta_{2} d r k h+e \theta_{2}{ }^{2} r^{2} h \\
& \left.+e \theta_{2} d r^{2}\right) /\left(k e\left(2 a^{2} d h k-2 r k a^{2} h-a d e h k+2 a r d+2 a h k e r-e r d\right)\right),
\end{aligned}
$$

where $\theta_{2}$ is the root of the equation

$$
\begin{aligned}
& \left(4 r h^{2} k a^{2}-6 h^{2} a k e r+h^{3} a^{2} k^{2} e+h r^{2} e+4 r e^{2} k h^{2}\right) Z^{3}+\left(d r^{2} e+4 r e^{2} k h d\right. \\
& \left.+2 r^{2} e^{2} k h+8 r d k a^{2} h-10 d a h k e r+2 r e^{2} k^{2} a h^{2}+d a^{2} h^{2} k^{2} e\right) Z^{2}+\left(r^{2} e^{2} k d\right. \\
& -h r^{2} k^{2} a^{2} e+2 r^{2} e^{2} k^{2} a h+4 r d^{2} k a^{2}-4 r e d^{2} k a+r e^{2} k d^{2} \\
& \left.+r e^{2} k^{2} a h d\right) Z+r^{2} e^{2} k^{2} a d-d r^{2} k^{2} a^{2} e=0
\end{aligned}
$$

$m^{[t b]}$ and $d=d^{[t b]}$ are given by

$$
\begin{aligned}
m^{[t b]}= & \left(4 a^{7} h^{5} \theta_{3} k^{5}-5 a^{6} h^{5} \theta_{3} k^{5} e+4 a^{6} h^{4} \theta_{3} k^{4} r-3 a^{5} h^{4} \theta_{3} k^{4} e r-2 a^{4} h^{4} e^{2} \theta_{3} k^{4} r\right. \\
& +a^{4} h^{3} \theta_{3} k^{3} r^{2} e+a^{3} h^{3} e^{2} \theta_{3} k^{3} r^{2}-a^{3} h^{2} \theta_{3} k^{2} e r^{3}+3 a^{2} h^{2} e^{2} r^{3} \theta_{3} k^{2}-a h e^{2} r^{4} \theta_{3} k \\
& -\theta_{3} e^{2} r^{5}-4 k^{4} a^{6} h^{4}+4 k^{4} e a^{5} h^{4}-4 k^{3} a^{5} h^{3} r+2 r k^{3} a^{4} h^{3} e+2 k^{3} e^{2} a^{3} h^{3} r+2 k a^{2} h e r^{3} \\
& \left.-2 k e^{2} a h r^{3}\right) /\left(( 4 a ^ { 3 } h ^ { 2 } k ^ { 2 } - a ^ { 2 } e h ^ { 2 } k ^ { 2 } + 2 a h k e r - r ^ { 2 } e ) \left(-2 a^{2} h k+4 a^{3} h^{2} \theta_{3} k^{2}+2 \theta_{3} k h\right.\right. \\
& \left.\left.-3 a^{2} \theta_{3} k^{2} h^{2} e+\theta_{3} r^{2} e+2 a e h k\right)\right),
\end{aligned}
$$




$$
\begin{aligned}
d^{[t b]}= & \left(a k h \left(-16 r k^{3} a^{4} h^{3} e-18 r^{2} k^{2} a^{2} h^{2} e^{2}+16 a^{7} h^{5} \theta_{3} k^{5}-12 r^{2} k^{2} a^{4} h^{2}+8 k^{3} a^{5} h^{3} r\right.\right. \\
& +11 k^{4} e a^{5} h^{4}+k^{4} e^{2} a^{4} h^{4}+3 \theta_{3} e^{2} r^{5}-12 k^{4} a^{6} h^{4}-a e r^{4}+e^{2} r^{4}-a^{5} h^{5} \theta_{3} k^{5} e^{2} \\
& -16 a^{6} h^{4} \theta_{3} k^{4} r+8 k^{3} e^{2} a^{3} h^{3} r+8 k e^{2} a h r^{3}-8 k a^{2} h e r^{3}+30 k^{2} a^{3} h^{2} e r^{2}+32 a^{5} h^{3} \theta_{3} k^{3} r^{2} \\
& -9 a^{4} h^{4} e^{2} \theta_{3} k^{4} r-26 a^{2} h^{2} e^{2} r^{3} \theta_{3} k^{2}+8 a^{2} h \theta_{3} k e r^{4}-56 a^{4} h^{3} \theta_{3} k^{3} r^{2} e+30 a^{3} h^{3} e^{2} \theta_{3} k^{3} r^{2} \\
& \left.+40 a^{3} h^{2} \theta_{3} k^{2} e r^{3}+3 a h e^{2} r^{4} \theta_{3} k+24 a^{5} h^{4} \theta_{3} k^{4} e r-16 a^{6} h^{5} \theta_{3} k^{5} e\right) /\left(\left(-4 a^{4} h^{2} k^{2}\right.\right. \\
& +8 a^{5} \theta_{3} k^{3} h^{3}+3 a^{3} e h^{2} k^{2}-6 a^{4} \theta_{3} k^{3} h^{3} e+a^{2} h^{2} e^{2} k^{2}-a^{3} h^{3} \theta_{3} k^{3} e^{2}+2 r a^{2} k e h-2 a h e^{2} r k \\
& +3 a^{2} h^{2} e^{2} \theta_{3} k^{2} r-r^{2} a e+6 a^{2} \theta_{3} k h e r^{2}+r^{2} e^{2}-3 a h e^{2} \theta_{3} k r^{2} \\
& \left.\left.+e^{2} r^{3} \theta_{3}\right)\left(4 a^{3} h^{2} k^{2}-a^{2} e h^{2} k^{2}+2 a h k e r-r^{2} e\right)\right),
\end{aligned}
$$

where $\theta_{3}$ is given by

$$
\theta_{3}=\frac{-a e h k+2 a^{2} h k+e r \pm \sqrt{a^{3} e h^{2} k^{2}+2 r a^{2} k e h+r^{2} a e}}{4 a^{3} h^{2} k^{2}-a^{2} e h^{2} k^{2}+2 a h k e r-r^{2} e} .
$$

Appendix A.2. Notations

The notations are same as that of [20]. We define $A^{2} F(U, U)$ and $A^{3} F(U, U, U)$ as follows: $A^{2} F(W, \lambda)(U, U)=\left(\begin{array}{l}\frac{\partial^{2} F_{1}}{\partial u^{2}} u_{1}^{2}+\frac{\partial^{2} F_{1}}{\partial u} u_{1} u_{2}+\frac{\partial^{2} F_{1}}{\partial v u} u_{2} u_{1}+\frac{\partial^{2} F_{1}}{\partial v^{2}} u_{2}^{2} \\ \frac{\partial^{2} F_{2}}{\partial u^{2}} u_{1}^{2}+\frac{\partial^{2} F_{2}}{\partial u \partial v} u_{1} u_{2}+\frac{\partial^{2} F_{2}}{\partial v \partial u} u_{2} u_{1}+\frac{\partial^{2} F_{2}}{\partial v^{2}} u_{2}^{2}\end{array}\right)$, and

$$
A^{3} F(W, \lambda)(U, U, U)=\left(\begin{array}{l}
\frac{\partial^{3} F_{1}}{\partial u^{3}} u_{1}^{3}+\frac{\partial^{3} F_{1}}{\partial u^{2} \partial v} u_{1}^{2} u_{2}+\frac{\partial^{3} F_{1}}{\partial v^{2} \partial u} u_{2}^{2} u_{1}+\frac{\partial^{3} F_{1}}{\partial v^{3}} u_{2}^{3} \\
\frac{\partial^{3} F_{2}}{\partial u^{3}} u_{1}^{3}+\frac{\partial^{3} F_{2}}{\partial u^{2} \partial v} u_{1}^{2} u_{2}+\frac{\partial^{3} F_{2}}{\partial v^{2} \partial u} u_{2}^{2} u_{1}+\frac{\partial^{3} F_{2}}{\partial v^{3}} u_{2}^{3}
\end{array}\right),
$$

where $U=\left(u_{1}, u_{2}\right)^{T}$ is the eigenvector corresponding to the matrix $J$.

Thus, at equilibrium $E_{2}=\left(u^{*}, v^{*}\right)$, where

$$
\begin{aligned}
u^{*} & =\psi_{1} k, \\
v^{*} & =-\frac{a k\left(e r^{2} \psi_{1}-2 r \psi_{1} m e+r \psi_{1} d a+2 m e r-a r d-r^{2} e-m^{2} e+d a m\right)}{\left(a^{2} h k+e r\right)\left(-r+r \psi_{1}+m\right)}(\mathrm{A} .2) \\
\psi_{1} & =\frac{r k a^{2} h-2 m e r+2 r^{2} e+a r d \pm \sqrt{\psi_{2}}}{2 r\left(a^{2} h k+e r\right)}, \\
\psi_{2} & =r^{2} k^{2} a^{4} h^{2}+4 r^{2} k a^{2} h m e-2 a^{3} d h k r^{2}+a^{2} d^{2} r^{2}-4 r k a^{2} h m^{2} e+4 r k a^{3} h d m,
\end{aligned}
$$


we have

$$
\begin{gathered}
\frac{\partial^{2} F_{1}}{\partial u^{2}}=\frac{-2 r}{k}+\frac{a m v^{*}}{\left(a u^{*}+v^{*}\right)^{2}}-\frac{a m v^{*}\left(a u^{*}-v^{*}\right)}{\left(a u^{*}+v^{*}\right)^{3}}, \quad \frac{\partial^{2} F_{1}}{\partial u \partial v}=-\frac{a m u^{*}}{\left(a u^{*}+v^{*}\right)^{2}}+\frac{a m u^{*}\left(a u^{*} 2-v^{*}\right)}{\left(a u^{*}+v^{*}\right)^{3}} \\
\frac{\partial^{2} F_{1}}{\partial u^{2}}=\frac{m u^{*}}{\left(a u^{*}+v^{*}\right)^{2}}+\frac{m u^{*}\left(a u^{*}-v^{*}\right)}{\left(a u^{*}+v^{*}\right)^{3}}, \quad \frac{\partial^{2} F_{2}}{\partial u^{2}}=-\frac{e a m v^{*}}{\left(a u^{*}+v^{*}\right)^{2}}+\frac{e a m v^{*}\left(a u^{*}-v^{*}\right)}{\left(a u^{*}+v^{*}\right)^{3}} \\
\frac{\partial^{2} F_{2}}{\partial u \partial y}=\frac{e a m u^{*}}{\left(a u^{*}+v^{*}\right)^{2}}-\frac{e a m u^{*}\left(a u^{*}-v^{*}\right)}{\left(a u^{*}+v^{*}\right)^{3}}, \quad \frac{\partial^{2} F_{2}}{\partial v^{2}}=-\frac{e m u^{*}}{\left(a u^{*}+v^{*}\right)^{2}}-\frac{e m u^{*}\left(a u^{*}-v^{*}\right)}{\left(a u^{*}+v^{*}\right)^{3}}-2 h \\
\frac{\partial^{3} F_{1}}{\partial u^{3}}=-\frac{2 a^{2} m v^{*}}{\left(a u^{*}+v^{*}\right)^{3}}+\frac{2 a^{2} m v^{*}\left(a u^{*}-2 v^{*}\right)}{\left(a u^{*}+v^{*}\right)^{4}}, \\
\frac{\partial^{3} F_{1} F_{1}}{\partial v^{2} \partial u}=-\frac{a m\left(a u^{*}-v^{*}\right)}{\left(a u^{*}+v^{*}\right)^{3}}+a m \frac{\left(a u^{*}-v^{*}\right)^{2}-2 a u^{*} v^{*}}{\left(a u^{*}+v^{*}\right)^{4}} \\
-\frac{m\left(a u^{*}-v^{*}\right)}{\left(a u^{*}+v^{*}\right)^{3}}-m \frac{\left(a u^{*}-v^{*}\right)^{2}-2 a u^{*} v^{*}}{\left(a u^{*}+v^{*}\right)^{4}}, \frac{\partial^{3} F_{1}}{\partial v^{3}}=-\frac{2 m u^{*}}{\left(a u^{*}+v^{*}\right)^{3}}+\frac{2 m u^{*}\left(a u^{*}-2 v^{*}\right)}{\left(a u^{*}+v^{*}\right)^{4}} \\
\frac{\partial^{3} F_{2}}{\partial u^{3}}=\frac{2 e a^{2} m v^{*}}{\left(a u^{*}+v^{*}\right)^{3}}-\frac{2 e a^{2} m v^{*}\left(a u^{*}-2 v^{*}\right)}{\left(a u^{*}+v^{*}\right)^{4}}, \\
\frac{\partial^{3} F_{2}}{\partial u^{2} \partial v}=-\frac{e a m\left(a u^{*}-v^{*}\right)}{\left(a u^{*}+v^{*}\right)^{3}}+e a m \frac{\left(a u^{*}-v^{*}\right)^{2}-2 a u^{*} v^{*}}{\left(a u^{*}+v^{*}\right)^{4}}
\end{gathered}
$$$$
\frac{\partial^{3} F_{2}}{\partial v^{2} \partial u}=e m \frac{\left(a u^{*}-v^{*}\right)}{\left(a u^{*}+v^{*}\right)^{3}}+e m \frac{\left(a u^{*}-v^{*}\right)^{2}-2 a u^{*} v^{*}}{\left(a u^{*}+v^{*}\right)^{4}}, \quad \frac{\partial^{3} F_{2}}{\partial v^{3}}=\frac{2 e m u^{*}}{\left(a u^{*}+v^{*}\right)^{3}}+\frac{2 e m u^{*}\left(2 a u^{*}-v^{*}\right)}{\left(a u^{*}+v^{*}\right)^{4}}
$$ 